\title{
Chicken interferon gamma fold expression level and tracheal lesion score differences between commercial broiler chickens vaccinated with live and vectored ILT vaccines
}

\author{
TOMAS MISKINIS, MARTIN LIMAN*, HENNING BISCHOFF*, VIDMANTAS BIZOKAS
}

Lithuanian University of Health Sciences, Tilžès g. 18, Kaunas, Lithuania

*AniCon Labor GmbH, Muehlenstraße 13, 49685 Hoeltinghausen, Germany

Miskinis T., Liman M., Bischoff H., Bizokas V.

Chicken interferon gamma fold expression level and tracheal lesion score differences between commercial broiler chickens vaccinated with live and vectored ILT vaccines

Summary

Infectious laryngotracheitis (ILT) is a respiratory tract disease affecting chickens around the world. The disease generates severe production losses due to increased mortality, decreased egg production, delayed body weight gain, and a predisposition to other respiratory pathogens causing enormous economic losses to the intensive poultry industry. Two licensed vaccines, comprising live or vectored ILT, are available to control the disease. The present trial was conducted to determine changes in chicken interferon gamma fold expression levels in chicken spleens and to evaluate tracheal lesion scores before and after vaccination with live and vectored infectious laryngotracheitis vaccines. Broilers were kept under commercial conditions until 35 days of age. Spleen and trachea samples were taken at 14, 28, and 35 days of age. Tracheas were stained with H\&E, histopathology was performed, and the INF- $\gamma$ fold expression level in spleen samples was analyzed. In the spleens of birds vaccinated with the live ILT vaccine, the increase in INF- $\gamma$ expression levels was statistically significantly $(p<0.01)$ lower at 28 days of age, or 7 days post vaccination, (trial 1$)$ and higher at 35 days of age, or 14 days post vaccination, (both trials). At 7 days post vaccination with the live ILT vaccine, the mean tracheal lesion score was significantly higher $(p<0.01)$ than it was in the control group (both trials). At 14 days post vaccination with the live ILT vaccine, the mean tracheal lesion score was significantly higher $(p<0.05)$ than it was in the control group 3 (trial 1). No significant differences were found between the control group 3 and group 2, which was vaccinated with the vectored HVT/LT vaccine (both trials). The results of the histology of trachea lesions indicate that vaccination with live ILT may have induced early local immunity. The INF- $\gamma$ analysis results might indicate that the birds in group 2 (HVT/LT) did not develop local immunity until 35 days of age From the practical point of view, the vectored ILT vaccine is more beneficial because of its ease of administration in the hatchery, lower labor cost, and the absence of clinical signs post vaccination. Its potential drawback, however, is poor local immunity and the slow onset of optimal immunity in commercial broiler chickens.

Keywords: Chicken INF- $\gamma$, ILT, HVT/LT, local immunity

Infectious laryngotracheitis virus (ILTV) is a member of the genus Iltovirus, family Herpesviridae, subfamily Alphaherpesvirinae. ILTV is taxonomically classified as Gallid herpesvirus 1 (6) and is the causative agent of a respiratory disease known as infectious laryngotracheitis in chickens. The disease occurs frequently in densely populated poultry production areas and generates severe production losses due to increased mortality, decreased egg production, delayed body weight gain, and a predisposition to other respiratory pathogens (12).
The disease is characterized by signs of respiratory depression, gasping, expectoration of bloody mucus, and high mortality (1). The clinical signs range from mild to severe, with mortality rates of up to $70 \%$, depending on the virulence of the infecting virus and management conditions (5).

The glycoproteins of ILTV are important protective antigens that can elicit both humoral and cell-mediated immunity (34). A subunit vaccine with the 205 kilodalton $(\mathrm{kDa})$ glycoprotein complex of ILTV containing glycoprotein $\mathrm{B}(\mathrm{gB})$ provided excellent protection 
against challenge (35). Glycoprotein B is highly conserved within all members of the Herpesviridae and is essential for herpesvirus infectivity because of its involvement in virus attachment and penetration (19, $25)$. It has also been shown that herpesvirus $\mathrm{gB}$ protein elicits neutralizing antibodies $(4,17)$ as well as cellmediated immune responses $(23,37)$. Therefore, it is considered to be an excellent candidate antigen for subunit or recombinant vaccines $(4,23)$. The ILTV gB possesses characteristics that are common to herpesvirus $\mathrm{gB}$, including conserved 10 cysteine residues on the surface of the molecule and conserved positions of N-linked glycosylation sites (9). It has been shown that the ILTV gB protein assembles into homodimers that are rapidly cleaved to form two disulphide-linked species (20). Recombinant fowlpox virus-vector vaccines expressing the ILTV gB gene, and a DNA vaccine containing the gB gene, have also been developed and have been shown to provide protective immunity in chickens $(3,7,26,30)$.

DNA-based vaccination offers an attractive alternative. It has been recognized, however, that DNA vaccines alone often generate only weak immune responses. Many studies have shown that the immunogenicity of an antigen can be enhanced by cytokines, including interleukin-2 (IL-2), interferon gamma (IFN- $\gamma$ ), IL-6, and IL-18 $(28,33,34,38)$.

IFN- $\gamma$ is the only member of type II IFN in birds and mammals and was postulated to serve as a bridge between innate and adaptive immunity. IFN- $\gamma$ plays a crucial role in regulating the maturation and differentiation process of several immune cells and activates $\mathrm{T}$ helper 1-type immune responses (8).

In two studies conducted by the same working group, it was found that chicken interferon gamma receptor type 1 (IFNGR1) was highly expressed in the spleen, thymus, peripheral blood lymphocytes (PBLs), cecal tonsil, lung, and liver, whereas chicken IFNGR2 was highly expressed in the spleen, thymus, PBLs, cecal tonsil, and muscle $(13,14)$.

Likewise, recent studies have clearly defined the antiviral role of IFN- $\gamma$ and its adjuvant properties against viruses of diverse genetic nature, including Newcastle disease virus (NDV), Marek's disease virus MDV, and influenza viruses $(15,24,27)$. Similar to its mammalian counterparts, chicken IFN- $\gamma$ also induces MHC class I and class II molecules and mediates the production of nitric oxide, which is an important inhibitory mechanism for viruses (16).

We hypothesized that classical vaccination with live infectious laryngotracheitis vaccine virus will induce a change in relative expression levels of chicken IFN- $\gamma$ in the spleen. We assumed that this effect will differ between live vaccinated, recombinant HVT/LT vaccinated and control groups. In addition, live ILTV vaccine replication may harm the tracheal epithelium because of local inflammation, which will not occur in recombinant vaccine and control groups.
An in vivo vaccination trial was conducted to determine changes in chicken IFN- $\gamma$ expression in the spleen at different time points and to evaluate microscopic changes in the tracheal epithelium in all trial groups.

\section{Material and methods}

In vivo vaccination trial design. The trial was performed in a standard commercial poultry farm, and 1500 male Ross 308 cross broilers were chosen. The broiler chickens were divided into three separate groups of 500 chicks each. Two subsequent identical trials 1 and 2 were made, each with 1500 male chicks. A different vaccination program was applied to each group, as shown in Table 1.

\section{Tab. 1. Broiler chicken vaccination program}

\begin{tabular}{|c|c|c|l|}
\hline Group & $\begin{array}{c}\text { Number } \\
\text { of chicks }\end{array}$ & $\begin{array}{r}\text { Vaccination } \\
\text { at day of age }\end{array}$ & \multicolumn{1}{|c|}{ Vaccine and application } \\
\hline \multirow{2}{*}{ No. 1 (live ILT) } & 500 & 1 & live HVT - subcutaneous \\
& & 1 & IB - spray \\
& 1 & ND - spray \\
live ILT - eye drop \\
No. 2 (rec. ILT) & 500 & 1 & rec. HVT/LT - subcutaneous \\
& & 1 & IB - spray \\
& & 1 & ND - spray \\
No. 3 (control) & 500 & 1 & live HVT - subcutaneous \\
& & 1 & IB - spray \\
& & ND - spray \\
\hline
\end{tabular}

Vaccine characteristics. Group 1 was vaccinated with a live infectious laryngotracheitis virus vaccine at 21 days of age via eye drop. The vaccine contains live Salsbury \# 146 strain of infectious laryngotracheitis virus with a titer of at least $10^{2.5}$ EID50.

In group 2, a recombinant viral vector vaccine HVT/LT was used at 1 day of age. This vaccine is based on Marek's disease vaccine strain serotype 3 (herpesvirus of turkeys/ HVT) expressing a key protective antigen $-\mathrm{gB}$ gene of infectious laryngotracheitis virus. A volume of $0.2 \mathrm{ml}$ of diluted vaccine solution, prepared according to the manufacturer's instructions, was injected subcutaneously into the neck of each broiler chicken. Group 3 was not vaccinated with any ILT vaccine and served as a control group.

In groups 1 and 3, Marek's disease vaccine (HVT serotype 3) was administered subcutaneously at 1 day of age. In all trial groups, an infectious bronchitis (IB) vaccine, containing H120 + D274 strains, and a Newcastle disease (ND) vaccine, containing VG/GA strains, were applied in the hatchery on the first day of chicks' life via coarse spray.

Spleen collection. From each trial group 10 birds aged 14, 28 and 35 days were taken. Birds were euthanized by cervical dislocation. Spleenectomy was performed. Spleens from each bird were placed individually in microcentrifuge tubes on dry ice. Within 3 hours the spleen samples were placed in $-80^{\circ} \mathrm{C}$. The samples were kept at $-80^{\circ} \mathrm{C}$ until all samples had been were collected, and then RNA extraction and RT-qPCR tests were performed.

H\&E staining and histopathology. To detect histopathological lesions, tracheas were collected, fixed in a $10 \%$ formalin buffer and stained using a standard haematoxylin and eosin (H\&E) protocol. Lesions were evaluated microscopically. Trachea lesion scores were determined and compared 
between groups. Lesions were scored by the Guy et al. (11) scoring method as follows:

Score 0 - normal. Epithelium consists of thin pseudostratified columnar epithelium. Mucous glands normal. Score 1 - mild changes. Normal epithelium with mild to moderate infiltration of lymphocytes, heterophils rare. Mucous glands normal. No syncytia or cells with intranuclear bodies. Score 2 - mild changes. Mucosa thickened because of mild to moderate cell infiltration and/or epithelium essentially normal except for foci of syncytia with intranuclear inclusion bodies. Hyperemia, occasionally with cell cuffs. Score 3 - moderate changes. Mucosa thickened because of moderate to marked cell infiltration. Numerous syncytia with intranuclear inclusion bodies. Patches of affected epithelium often separating from or, less commonly, sloughed from lamina propria. Mucosal surface well covered by normal or affected epithelium. Mucous glands reduced. Marked hyperemia, cuffs of mononuclear cells around vessels outside mucosa. Score 4 - Severe changes. Mucosa thickened because of edema, proteinaceous fluid, cellular exudate, or adherent fibrinohemorrhagic to cellular pseudomembrane on the surface. Normal epithelium absent, the mucosal surface covered by a thin layer of basal cells. Syncytia with inclusion bodies sometimes present. Score 5 - Very severe changes. The same as 4 , except that mucosa has no residual epithelium and syncytia with inclusion bodies are rarely found.

Feed and water. All groups were fed ad libitum using hand feeders. Water consumption was not limited. The number of feeders and drinkers was calculated according to the animal welfare requirements.

INF- $\gamma$ expression analysis. Frozen spleen samples were transferred directly from $-80^{\circ} \mathrm{C}$ into $1 \mathrm{ml}$ of RNAlater solution (Thermo Fisher Scientific, MA, USA) and incubated at $4{ }^{\circ} \mathrm{C}$ overnight. The organs were then transferred into $15 \mathrm{ml}$ round-bottom tubes containing $2 \mathrm{ml}$ of RNase/DNase-free water and two $5 \mathrm{~mm}$ metal beads. The samples were subsequently homogenized in an MP FastPrep-24 system and spinned down briefly. RNA was isolated from $75 \mu \mathrm{l}$ of homogenized sample supernatant using an RNeasy kit (Qiagen, Hilden, Germany). RNA concentrations were determined in a NanoDrop ${ }^{\circledR}$ ND1000 Spectrophotometer (PeqLab, Erlangen, Germany). RNA integrity was determined using a QIAxcel system (Qiagen, Hilden, Germany).

IFN- $\gamma$ specific primers and probe were used according to Tippenhauer et al. (29) to detect a partial IFN- $\gamma$ gene sequence (NCBI Gene ID 396054). The RPL13 specific primers and probe were used according to Mitra et al. (18) to detect the housekeeping reference gene of chicken ribosomal protein L13 (Accession Number NM 204999). Synthesis of cDNA was carried out in duplicates with $250 \mathrm{ng}$ of total RNA using qScript cDNA Supermix (Quantabio, MA, USA). Both qPCR reactions were carried out using BCD 2x qPCR Mix (AniCon, Hoeltinghausen, Germany) according to the manufacturer's recommendations, resulting in final concentrations of both primers $(600 \mathrm{nM}$ for IFN- $\gamma$ and $400 \mathrm{nM}$ for RPL13) and probes (200 nM each). Data were collected using a CFX384 Real-Time PCR thermal cycler (BioRad). The standard curve for IFN- $\gamma$ was based on the dilution of plasmid DNA. The RPL13 standard curve was based on total RNA isolated from chicken spleens. Each cDNA sample was tested in duplicate. Non-template controls and $\log 10$ dilution series of standards were included in each run. No reverse transcription controls were conducted for every sample to rule out contamination by genomic DNA.

Quantitation was done by the $\Delta \Delta \mathrm{Ct}$ method, according to MIQE guidelines (2). Briefly, the detected reporter fluorescence was normalized and then standardized with respect to the corresponding RPL13 rRNA level as an indicator of homology in the RNA input. Outliers were identified using Grubb's test $(p<0.05)$ and excluded from the analysis. The data are presented as the average mRNA fold change based on the calculated corrected cycle threshold (CT)

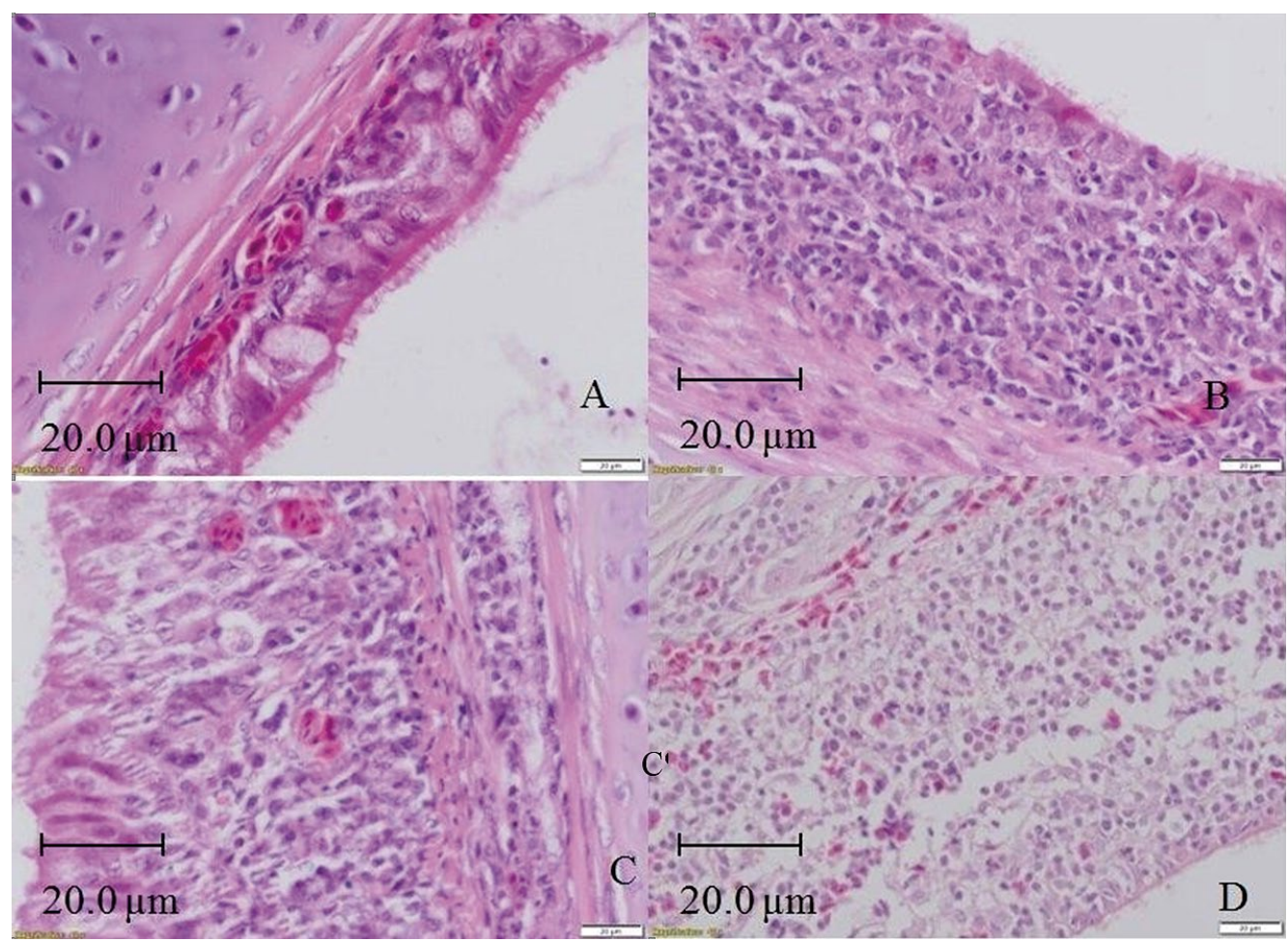

Fig. 1. Representative microscopical images of tracheal lesion scores (magnification $40 \times$ ) Explanations: A: Score 0 - Normal. Epithelium consists of thin pseudostratified columnar epithelium. Mucous glands normal. B: score 1 - Mild changes. Normal epithelium with mild to moderate infiltration of lymphocytes, heterophils rare. Mucous glands normal. No syncytia or cells with intranuclear bodies. C: score 2 - Mild changes. Mucosa thickened because of mild to moderate cell infiltration and/or epithelium essentially normal except for foci of syncytia with intranuclear inclusion bodies. Hyperemia, occasionally with cell cuffs. D: score 3 - Moderate changes. Mucosa thickened because of moderate to marked cell infiltration. 
values. Arithmetic means were determined per group and day of age.

Data analysis. Data analysis was performed using the Bio-Rad CFX Manager software, GraphPad Prism software and MS Excel 2016 software. Statistical analysis was performed using the ANOVA test.

\section{Results and discussion}

Evaluation of tracheal lesion score. The mean tracheal lesion score was determined at 14, 28 and 35 days of age. Representative microscopic images of tracheal lesion scores are shown in Figure 1.

We found significant differences at 28 and 35 days of age in group 1 in comparison with group 3 (the control group) in both trials. At 28 days of age, the mean tracheal lesion score was statistically significantly higher $(\mathrm{p}<0.001)$ in group 1 than it was in group 3. At 35 days of age the mean tracheal lesion score in group 1 was statistically significantly higher $(p<0.001)$ than it was in group 3. Compared with groups 1 and 3, no significant differences were determined in group 2, which was vaccinated with the vectored HVT/ LT vaccine.

Mean tracheal lesion scores at different broiler ages are shown in Figure 2.

These changes occurred at 28 days of age in group 1, in which the live ILTV vaccine was applied at 21 days of age via eye drop. Tracheal lesions were less severe at 35 days of age than they were at 28 days of age.

The tracheal count of different lesion scores at 28 and 35 days of age in trials 1 and 2 are shown separately in Figures 3 and 4. As shown in Figures 3 and 4, 7 tracheas with score 2 and 3 tracheas with score 3 were found during trial 1 in group 1 at 28 days of age. At 35 days of age, 3 tracheas with score 1 and 2 tracheas with score 2 were found. These findings were significantly different from those in group 2, which was vaccinated with the vectored $\mathrm{HVT} / \mathrm{LT}$ vaccine, and 1 and 2 in group 3, which was not vaccinated. In both group 2 and group $3,90 \%$ of the tracheas had score 0 and no lesions.

Chicken INF- $\gamma$ expression levels in spleens at different time points. Chicken INF- $\gamma$ expression levels in the spleen at 14, 28, and 35 days of age were determined. No significant differences were found between the trial groups at 14 days of age. Statistically significant changes were seen at 28 and 35 days of age. In comparison with the non-vaccinated group 3, a significant $(\mathrm{p}<0.01)$ decrease in IFN- $\gamma$ was seen at 28 days of age (trial 1$)$ and a significant $(\mathrm{p}<0.001)$ increase in IFN- $\gamma$ was seen at 35 days of age (both
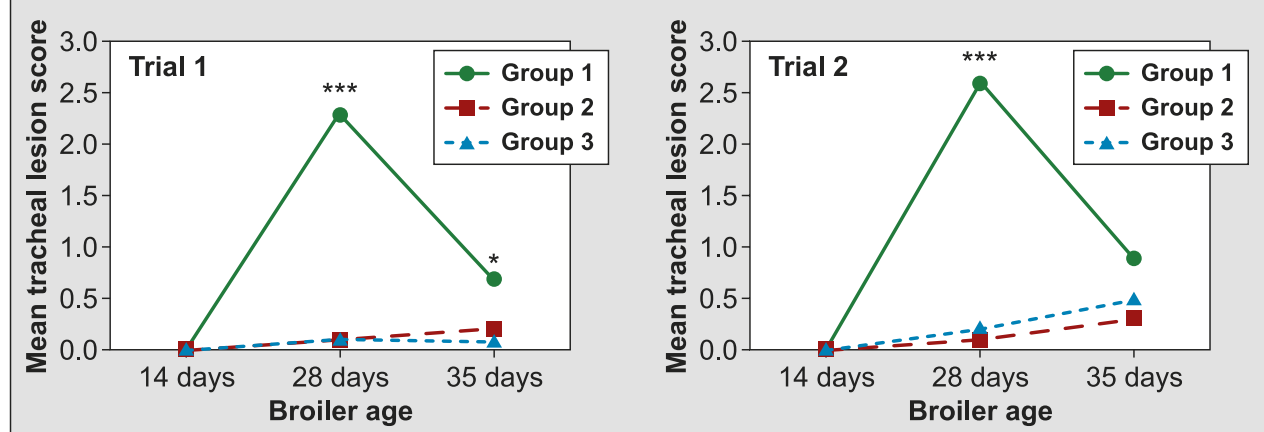

Fig. 2. Mean tracheal lesion score at different time points

Explanations: At 28 days of age, the mean tracheal lesion score was statistically significantly higher $\left(\mathrm{p} \leq 0.001\right.$, where $\left.* * *-p \leq 0.001 ; * *-\mathrm{p} \leq 0.01 ; *_{-}^{*} \mathrm{p} \leq 0.05\right)$ in group 1 than it was in group 3 in both trials. This difference was less prominent after 35 days, when a significant difference $(\mathrm{p} \leq 0.05)$ was observed between group 1 and group 3 only in trial 1 .

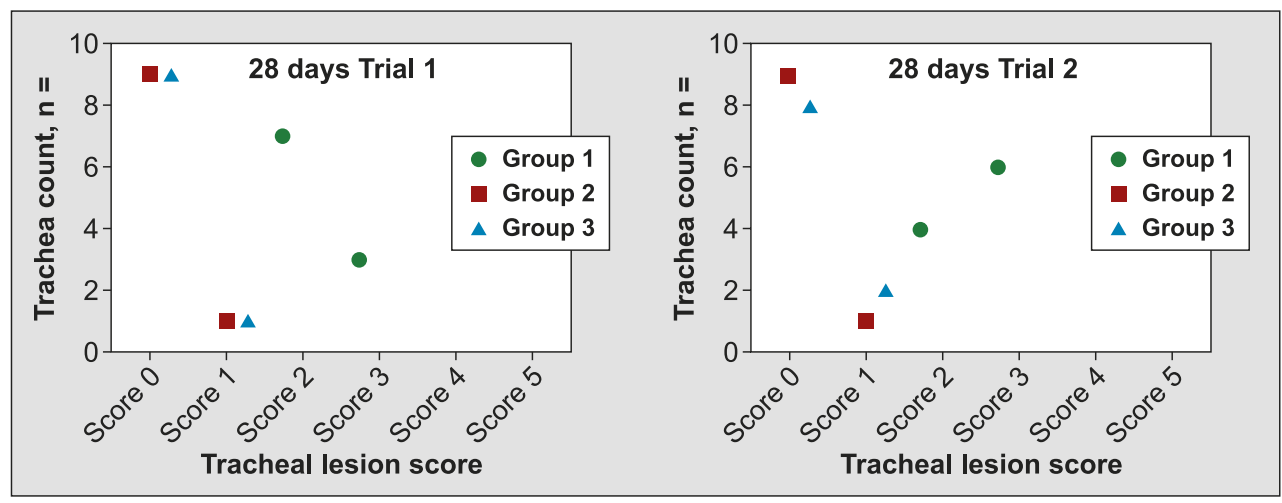

Fig. 3. Data show tracheal counts per lesion score at 28 days of age separately for trials 1 and 2 . The most severe changes are seen in both trials in group 1 , which was vaccinated with the live ILT vaccine

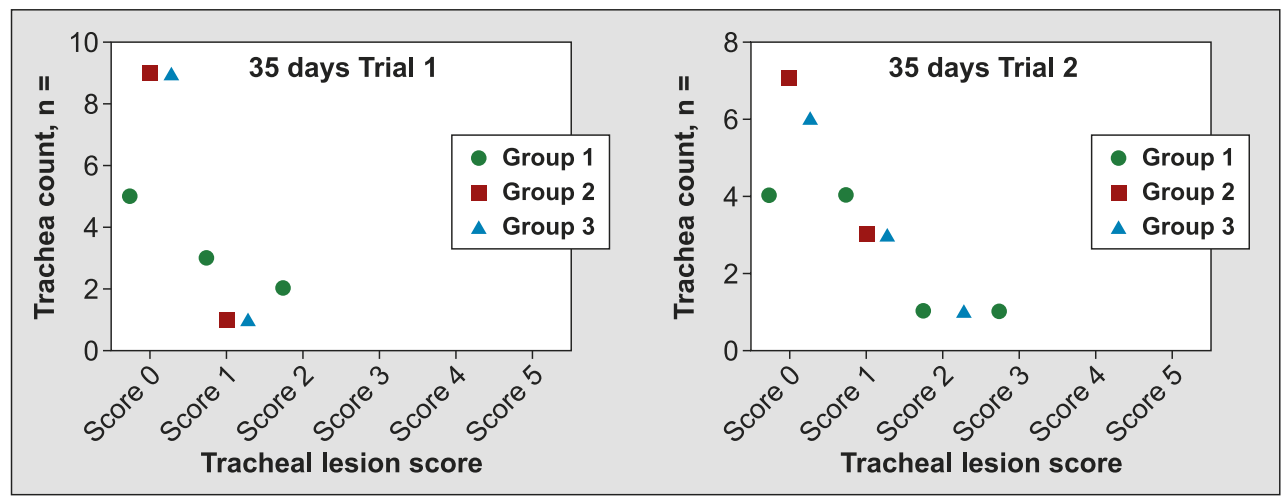

Fig. 4. Data show tracheal counts per lesion score at 35 days of age separately for trials 


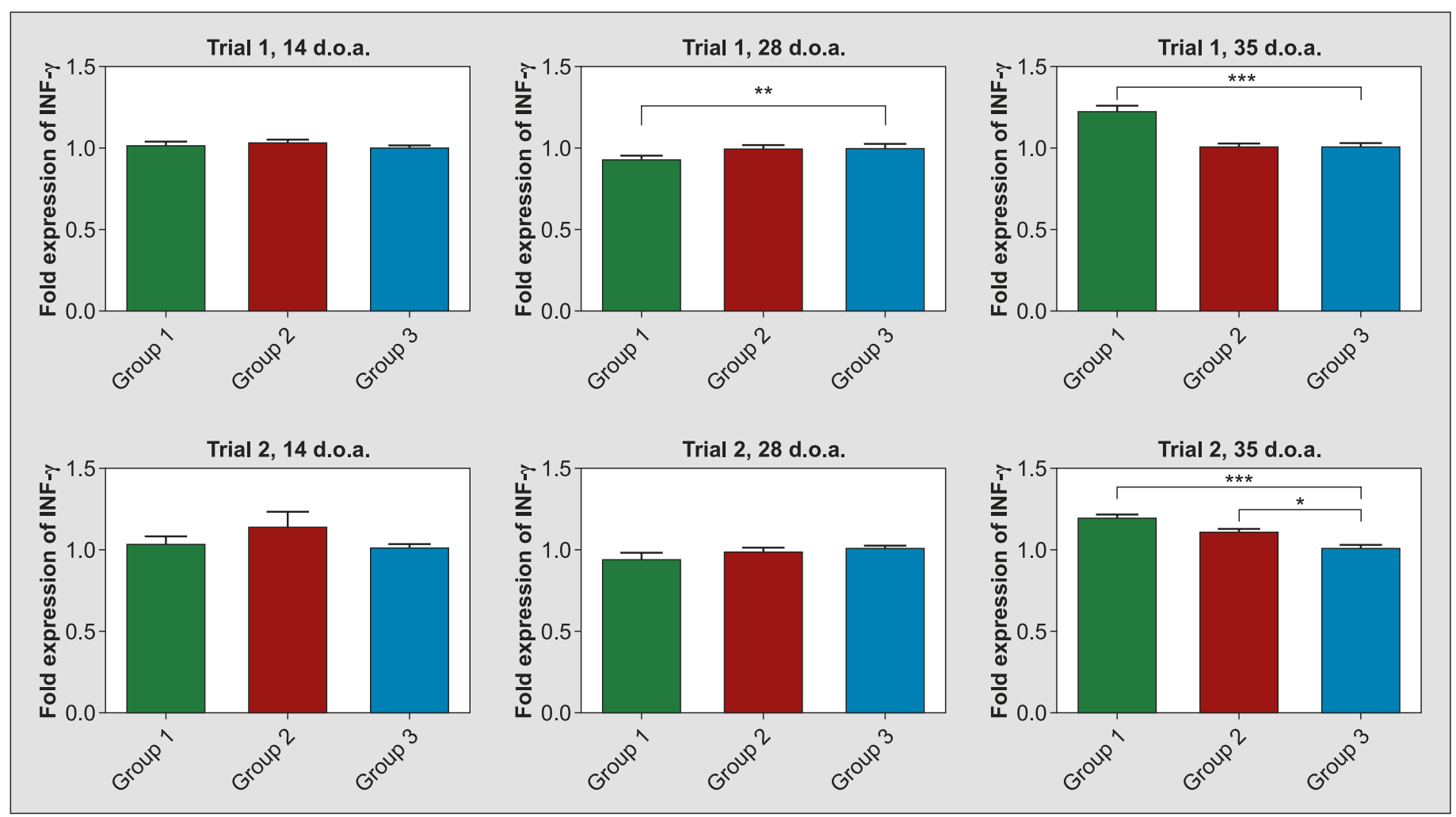

Fig. 5. Fold expression of INF- $\gamma$ mRNA levels in spleens at different time points

Explanations: Statistical significance (where $* * *-p \leq 0.001 ; * *-p \leq 0.01 ; *-p \leq 0.05$ ) is illustrated by bricks. Animals were euthanized, and total RNA was isolated from their spleens. Isolated RNA was subjected to cDNA synthesis, subsequent qPCR, and analyses of relative expression levels according to the $\Delta \Delta \mathrm{Ct}$ method.

trials) in group 1, which was vaccinated with the live ILTV vaccine. In group 2, which was vaccinated with the vectored HVT/LT vaccine, no significant changes were seen in trail 1 but seen only in trail 2 at 35 days of age $(p<0.05)$, when compared to the control group. The levels of the relative fold expression of INF- $\gamma$ mRNA at different time points are shown in Figure 5.

Outbreaks of infectious laryngotracheitis produce severe economic losses due to high mortality and poor performance. In some ILT enzootic areas where where high infectious pressure from field virus is present and an early infection is possible, complex vaccination strategies, such as live + vectored ILT, are recommended.

Vagnozzi et al. (32) found that immediately after challenge with ILTV a significant increase in IFN- $\gamma$ gene expression was followed by a significant reduction in viral replication. In contrast to the rapid induction of IFN- $\gamma$, the expression of pro-inflammatory cytokines (IL-1 $\beta$, IL-6, IL-8) and type I IFN- $\beta$ was either slightly reduced or remained at basal levels. This suggests that the former cytokines may not play important roles in immediate early responses induced by ILTV challenge in either vaccinated or non-vaccinated chickens. Overall, these results suggest that the rapid expression of IFN- $\gamma$ may induce pathways of antiviral responses necessary for blocking early virus replication. In our study, we found a significant $(\mathrm{p}<0.05)$ increase in INF- $\gamma 14$ days after vaccination with the live ILT vaccine. Indeed, the results of the histology of trachea lesions indicate that the vaccination with live ILT may have induced early local immunity and may have helped to protect young birds.

We found significant differences at 28 and 35 days of age in group 1 in comparison with the control group 3 in both trials. At 28 days of age, the mean tracheal lesion score was statistically significantly higher $(p<0.001)$ in group 1 than it was in group 3. At 35 days of age, the mean tracheal lesion score in group 1 was statistically significantly higher $(\mathrm{p}<0.001)$ than it was in group 3. Compared with groups 1 and 3, no significant differences were found in group 2, which was vaccinated with the vectored HVT/LT vaccine (both trials). The live vaccine caused local inflammation in the tracheal epithelium. Microscopically, the epithelium in some tracheas was almost regenerated at 35 days of age. Based on a study by Vagnozzi et al. (32) and other observations, it is speculated that ILTV viral vector vaccines can mitigate the disease to a certain degree. However, due to their inability to reduce challenge virus replication in the trachea, they may not sufficiently reduce the circulation of virulent strains in areas of overwhelming field challenge, where they have failed to contain outbreaks.

Purcell (21) and Guy and Garcia (10) have shown that within $72 \mathrm{~h}$ of experimental infection, there is a mild infiltration of small numbers of lymphocytes and polymorphonuclear cells (PMN, presumably het- 
erophils) in the lamina propria in areas where syncytia are present. Between days 3 and 5 post infection, there is a more severe edema of the lamina propria and underlying tissues, with numerous macrophages and lymphocytes (21), as well as histiocytes and plasma cells (12), present throughout the lamina propria, and with PMNs infiltrating edematous areas around capillaries and migrating in larger numbers to the hyperplastic epithelium (21).

Rauw et al. (22) measured chicken INF- $\gamma$ by the capture ELISA and found that cell-mediated immune response was higher in a vectored HVT-ND/live NDtreated group compared with immune response in groups that were non-vaccinated or vaccinated with a vectored vaccine only. In the present trial, we found that the expression levels of INF- $\gamma$ in the spleens of birds vaccinated with the live infectious laryngotracheitis virus vaccine were statistically significantly $(\mathrm{p}<0.01)$ lower at 28 days of age, or 7 days post vaccination, (trial 1) and significantly $(p<0.001)$ higher at 35 days of age, or 14 days post vaccination, (both trials). This is probably due to the reaction to the virus, such as local inflammation in the trachea after vaccination. In group 2, which was vaccinated with the vectored HVT/LT vaccine, no significant changes were seen in trail 1 but seen only in trail 2 at 35 days of age $(\mathrm{p}<0.05)$, when compared to the control group. An explanation for this may be that the vectored vaccine has no clinical effect on the airways, and thus it may have less capacity to induce inflammation. It might also indicate that during the trial period the birds in group 2 did not develop local immunity until 35 days of age.

A method for analyzing chicken INF- $\gamma$ expression could be useful in evaluating and assessing local immunity, since in the present study of the spleen significant differences $(p<0.05)$ in the fold INF- $\gamma$ expression level were found between the live and vectored ILT vaccinated groups compared with the non-vaccinated group. Further trials are required to compare different local immunity assessment methods, such as cell-derived antibodies (CD8), the ciliostasis test, and others.

However, chicken INF- $\gamma$ expression needs to be evaluated further and in different organs, such as the bursa of Fabricius. A decrease in INF- $\gamma$ expression levels could also be associated with immunosuppressive agents, such as Marek's disease virus and Newcastle disease virus.

Further investigations can be performed to evaluate the duration of cell-mediated immune response in long living birds such as broiler breeders or layers using different ILT vaccination strategies and vaccine administration methods.

It is also worth noting that the tracheal epithelium recovered in most birds only two weeks after vaccination with the live infectious laryngotracheitis vaccine. It is therefore important in making a vaccination schedule for broiler chickens to allow a proper time lag between live respiratory disease vaccinations that target the same upper respiratory tract cells or induce local respiratory immunity.

From the practical point of view, vaccination with the vectored ILT vaccine in commercial chicken broiler production is more beneficial because of its ease of administration in the hatchery, lower labor cost, and the absence of clinical signs post vaccination. A potential drawback is the late onset of immunity and poor local immunity at an early age. Vagnozzi et al. (31) also showed that immunity against ILTV generated by recombinant vaccines may take more than a month to develop.

\section{References}

1. Bagust T. J., Guy J. S.: Laryngotracheitis, [in:] Calnek B. W. (ed.): Disease of Poultry. Iowa State University Press, Ames 1997, 527-539.

2.Bustin S. A., Benes V., Garson J. A., Hellemans J., Huggett J., Kubista M., Mueller R., Nolan T., Pfaff M. W., Shipley G. L., Vandesompele J., Wittwer C. T.: The MIQE guidelines: minimum information for publication of quantitative real-time PCR experiments. Clin. Chemistry 2009, 55, 611-622.

3. Chen H. Y., Cui P., Cui B. A., Li H. P., Jiao X. Q., Zheng L. L., Cheng G., Chao $A$. J.: Immune responses of chickens inoculated with a recombinant fowlpox vaccine coexpressing glycoprotein $B$ of infectious laryngotracheitis virus and chicken IL-18. FEMS Immuno. Med. Microbiol. 2011, 63, 289-295.

4. Cranage M. P., Kouzarides T., Bankier A. T., Satchwell S., Weston K., Tomlinson P., Barrell B., Hart H., Bell S. E., Minson A. C.: Identification of the human cytomegalovirus glycoprotein $B$ gene and induction of neutralizing antibodies via its expression in recombinant vaccinia virus. EMBO J. 1986, 5, 3057-3063.

5. Davidson I., Nagar S., Ribshtein I., Shkoda I., Perk S., Garcia M.: Detection of wild- and vaccine-type avian infectious laryngotracheitis virus in clinical samples and feather shafts of commercial chickens. Avian Dis. 2009, 53, 618-623.

6. Davison A. J., Eberle R., Hayward G. S., McGeoch D. J., Minson A. C., Pellet P. E., Roizman B., Studdert M. J., Thiry E.: The order Herpesvirales. Arch. Virology 2009, 154, 171-177.

7. Davison S., Gingerich E. N., Casavant S., Eckroade R. J.: Evaluation of the efficacy of a live fowlpox-vectored infectious laryngotracheitis/avian encephalomyelitis vaccine against ILT viral challenge. Avian Dis. 2006, 50, 50-54.

8. Fensterl V., Sen G. C.: Interferons and viral infections. Biofactors 2009, 35, 14-20.

9. Griffin A. M.: The nucleotide sequence of the glycoprotein gB gene of infectious laryngotracheitis virus: analysis and evolutionary relationship to the homologous gene from other herpesviruses. J. Gen. Virology 1991, 72, 393-398.

10. Guy J., García M.: Laryngotracheitis, [in:] Saif Y. M., Fadly A. M., Glisson J. R., McDougald L. L., Nolan L. K., Swayne D. E.: Diseases of Poultry, Blackwell Publishing, Ames 2008, 137-152.

11. Guy J. S., Barnes H. J., Morgan L. M.: Virulence of infectious laryngotracheitis viruses: comparison of modified-live vaccine viruses and North Carolina field isolates. Avian Dis. 1990, 34, 106-113.

12. Guy J. S., Garcia M.: Laryngotracheitis, [in:] Saif Y. M., Fadly A. M., Glisson J. R., McDougald L. R., Nolan L. K., Swayn D. E.: Diseases of Poultry, $12^{\text {th }}$ edition, Blackwell Publishing, Ames, Iowa, USA 2008, p. 137-152.

13. Han C. L., Zhang W., Dong H. T., Han X., Wang M.: A novel gene of beta chain of the IFN-gamma receptor of Huiyang chicken: cloning, distribution, and CD assay. J. Interferon Cytokine Res. 2006, 26, 441-448.

14. Han X., Chen T., Wang M.: Molecular cloning and characterization of chicken interferon-gamma receptor alpha-chain. J. Interferon Cytokine Res. 2007, 28, 445-454.

15. Haq K., Wootton S. K., Barjesteh N., Golovan S., Bendall A., Sharif S.: Effects of interferon-gamma knockdown on vaccine-induced immunity against Marek's disease in chickens. Canad. J. Vet. Res. 2015, 79, 1-7.

16. Mallick A. I., Parvizi P., Read L. R., Nagy E., Behboudi S., Sharif S.: Enhancement of immunogenicity of a virosome-based avian influenza vaccine in chickens by incorporating CpG-ODN. Vaccine 2011, 29, 1657-1665.

17. Marlin S. D., Highlander S. L., Holland T. C., Levine M., Glorioso J. C. Antigenic variation (mar mutations) in herpes simplex virus glycoprotein $\mathrm{B}$ can induce temperature-dependent alterations in $\mathrm{gB}$ processing and virus production. J. Virology 1986, 59, 142-153. 
18. Mitra T., Bilic I., Hess M., Liebhart D.: The 60 S ribosomal protein L13 is the most preferable reference gene to investigate gene expression in selected organs from turkeys and chickens, in context of different infection model. Veterinary Research 2016, 47, 105.

19. Pereira $L$ : Function of glycoprotein B homologues of the family Herpesviridae. Infect. Agents Dis. 1994, 3, 9-28.

20. Poulsen D. J., Keeler Jr C. L.: Characterization of the assembly and processing of infectious laryngotracheitis virus glycoprotein B. J. General Virol. 1997, 78, 2945-2951

21. Purcell $D$. A.: The ultrastructural changes produced by infectious laryngotracheitis virus in tracheal epithelium of the fowl. Res. Vet. Sci. 1971, 12 455-458

22. Rauw F., Gardin Y., Palya V., Anbari S., Lemaire S., Boschmans M., Berg T., Lambrecht $B$.: Improved vaccination against Newcastle disease by an in ovo recombinant HVT-ND combined with an adjuvanted live vaccine at day-old Vaccine 2009, 28, 823-833.

23. Sabbaj S., Pass R. F., Goepfert P. A., Pichon A.: Glycoprotein B vaccine is capable of boosting both antibody and CD4 T-cell responses to cytomegalovirus in chronically infected women. J. Infect. Dis. 2011, 203, 1534-1541.

24. Sawant P. M., Verma P. C., Subudhi P. K., Chaturvedi U., Singh M., Kumar R. Immunomodulation of bivalent Newcastle disease DNA vaccine induced immune response by co-delivery of chicken IFN-gamma and IL-4 genes. Vet. Immunol. Immunopathol. 2011, 144, 36-44.

25. Spear P. G.: Entry of alphaherpesviruses into cells. Seminars in Virology, 1993, 4, 167-180.

26. Sun H. L., Wang Y. F., Tong G. Z., Zhang P. J., Miao D. Y., Zhi H. D., Wang M. Wang M.: Protection of chickens from Newcastle disease and infectious laryngotracheitis with a recombinant fowlpox virus coexpressing the F, HN genes of Newcastle disease virus and $\mathrm{gB}$ gene of infectious laryngotracheitis virus. Avian Dis. 2008, 52, 111-117.

27. Susta L., Cornax I., Diel D. G., Garcia S. C., Miller P. J., Liu X.: Expression of interferon gamma by a highly virulent strain of Newcastle disease virus decreases its pathogenicity in chickens. Microb. Pathogen. 2013, 61-62, 73-83.

28. Tang M. J., Wang H. N., Zhou S., Tian G. B.: Enhancement of the immunogenicity of an infectious bronchitis virus DNA vaccine by a bicistronic plasmid encoding nucleocapsid protein and interleukin-2. J. Virol. Methods 2008, 149 , 42-48.

29. Tippenhauer M., Heller D. E., Weigend S., Rautenschlein S.: The host genotype influences infectious bursal disease virus pathogenesis in chickens by modulation of T cells responses and cytokine gene expression. Dev. Comp. Immunol. 2013, 40, 1-10.

30. Tong G. Z., Zhang S. J., Wang L., Qiu H. J., Wang Y. F., Wang M.: Protection of chickens from infectious laryngotracheitis with a recombinant fowlpox virus expressing glycoprotein B of infectious laryngotracheitis virus. Avian Pathol. 2001, 30, 143-148.

31. Vagnozzi A., Riblet S., Zavala G., Ecco R., Afonso C. L., Garcia M.: Evaluation of the transcriptional status of host cytokines and viral genes in the trachea of vaccinated and non-vaccinated chickens after challenge with the infectious laryngotracheitis virus. Avian Pathol. 2016, 45, 106-113.

32. Vagnozzi A., Zavala G., Riblet S. M., Mundt A., García M.: Protection induced by commercially available live-attenuated and recombinant viral vector vaccines against infectious laryngotracheitis virus in broiler chickens. Avian Pathol. 2012, 41, 21-31.

33. Yin R. L., Li C., Yang Z. T., Zhang Y. J., Bai W. L., Li X., Yin R. H., Liu H. Liu $S$.: Construction and immunogenicity of a DNA vaccine containing clumping factor A of Staphylococcus aureus and bovine IL18. Vet. Immunol. Immunopathol. 2009, 132, 270-274.

34. Yoon H. A., Aleyas A. G., George J. A., Park S. O., Han Y. W., Lee J. H., Kang H. Y., Kang S. H., Cho J. G., Eo S. K.: Modulation of immune responses induced by DNA vaccine expressing glycoprotein B of pseudorabies virus via coadministration of IFN-c-associated cytokines. J. Interferon Cytokine Res. 2006, 26, 730-738

35. York J. J., Fahey $K$. J.: Humoral and cell mediated immune responses to the glycoproteins of infectious laryngotracheitis herpesvirus. Arch. Virology 1990, 115, 289-297.

36. York J. J., Fahey K. J.: Vaccination with affinity-purified glycoproteins protects chickens against infectious laryngotracheitis herpesvirus. Avian Pathol. 1991, 20, 693-704.

37. Zarling J. M., Moran P. A., Burke R. L., Pachl C., Berman P. W., Lasky L. A. Human cytotoxic $\mathrm{T}$ cell clones directed against herpes simplex virus-infected cells. IV. Recognition and activation by cloned glycoproteins $\mathrm{gB}$ and $\mathrm{gD}$. J. Immunol. 1986, 136, 4669-4673.

38. Zhang H., Cheng C., Zheng M., Chen J. L., Meng M. J., Zhao Z. Z., Chen Q., Xie Z., Li J. L.: Enhancement of immunity to an Escherichia coli vaccine in mice orally inoculated with a fusion gene encoding porcine interleukin 4 and 6 . Vaccine 2007, 25, 7094-7101.

Corresponding author: Martin Liman, AniCon Labor GmbH, Muehlenstraße 13, 49685 Hoeltinghausen, Germany; e-mail: info@anicon.de 